\title{
SUBSPACES OF DE BRANGES SPACES WITH PRESCRIBED GROWTH
}

\author{
A. BARANOV AND H. WORACEK
}

\begin{abstract}
The growth properties of de Branges spaces and their subspaces are studied. It is shown that, for each given pair of growth functions $\lambda(r)=O(r)$ and $\lambda_{1}=o(\lambda)$, there exist de Branges spaces of growth $\lambda$ that have a de Branges subspace of growth $\lambda_{1}$. This phenomenon cannot occur for a class of de Branges spaces that, in a certain sense, behave regularly along the real axis.
\end{abstract}

\section{$\S 1$. INTRODUCTION}

A de Branges space is a Hilbert space $\langle\mathcal{H},(\cdot, \cdot)\rangle$ with the following properties:

(dB1) The elements of $\mathcal{H}$ are entire functions, and for each $w \in \mathbb{C}$ the point evaluation $F \mapsto F(w)$ is a continuous linear functional on $\mathcal{H}$.

(dB2) If $F \in \mathcal{H}$, then $F^{\#}(z):=\overline{F(\bar{z})}$ also belongs to $\mathcal{H}$, and $\left\|F^{\#}\right\|=\|F\|$.

(dB3) If $w \in \mathbb{C} \backslash \mathbb{R}$ and $F \in \mathcal{H}, F(w)=0$, then

$$
\frac{z-\bar{w}}{z-w} F(z) \in \mathcal{H} \quad \text { and } \quad\left\|\frac{z-\bar{w}}{z-w} F(z)\right\|=\|F\| \text {. }
$$

The theory of such Hilbert spaces of entire functions was founded by L. de Branges (cf. $[\mathrm{dB}]$ ). It was further developed by many authors (see, e.g., [B1, B2, GM, KWW2, $\mathrm{KW} 1, \mathrm{KW} 3, \mathrm{RR}]$ ) and found applications in various contexts (see, e.g., [BP1, DK1, DK2, Li, OS, Re, PW]).

Throughout this paper it is assumed that a de Branges space is additionally subject to the condition

(Z) For every $t \in \mathbb{R}$ there exists $F \in \mathcal{H}$ with $F(t) \neq 0$.

In most respects, this assumption causes no loss of generality (cf. [dB, Problem 44] and [KW3, Lemma 2.4]).

Prominent examples of de Branges spaces are the so-called Paley-Wiener spaces. For a real positive number $a$, let $\mathcal{P} \mathcal{W}_{a}$ be the set of all entire functions of exponential type at most $a$ whose restriction to $\mathbb{R}$ belongs to $L^{2}(\mathbb{R})$. Endowed with the norm $\|F\|:=$ $\left\|\left.F\right|_{\mathbb{R}}\right\|_{L^{2}(\mathbb{R})}$, the space $\mathcal{P} \mathcal{W}_{a}$ becomes a de Branges space.

In complex analysis, in particular in the theory of entire functions, the notion of growth plays a central role. A function $\lambda: \mathbb{R}^{+} \rightarrow \mathbb{R}^{+}$is called a growth function if it satisfies the following axioms:

(gf1) The limit $\rho:=\lim _{r \rightarrow \infty} \frac{\log \lambda(r)}{\log r}$ exists and is a finite nonnegative number.

(gf2) For all sufficiently large values of $r$, the function $\lambda$ is differentiable, and $\lim _{r \rightarrow \infty} r \frac{\lambda^{\prime}(r)}{\lambda(r)}$ $=\rho$.

(gf3) $\log r=o(\lambda(r))$.

2000 Mathematics Subject Classification. Primary 46E20; Secondary 46E22, 30D15.

Key words and phrases. de Branges space, growth function, de Branges subspace. 
Conditions (gf1) and (gf2) ensure that Valiron's theory of proximate orders is available, cf. [L, I.12] or [LG, I.6], as well as the theory of value distribution of meromorphic functions; cf. $[\mathrm{Ru}]$. Condition (gf3), saying that $\lambda$ grows sufficiently rapidly, is imposed to exclude trivial cases and is not an essential restriction. Unless specified, "O" - and " $O$ "-relations are always understood for $r \rightarrow \infty$.

Classical examples of growth functions are presented by functions of the form

$$
\lambda(r)=r^{\alpha}(\log r)^{\beta},
$$

where $\alpha, \beta \in \mathbb{R}, \alpha>0$.

Since the elements of a de Branges space are entire functions, it is a natural task to bring together the concepts of a de Branges space and a growth function, i.e., to study de Branges spaces from the viewpoint of growth properties of their elements. For general growth functions, a systematic study was initiated in [KW3]. For the particular case of exponential growth, i.e., $\lambda(r)=r$, such investigations go back to the very beginning of the theory of de Branges spaces (see, e.g., $[\mathrm{dB}]$ ).

Let us briefly describe the main theme of our present work. If $F$ is an entire function and $\lambda$ is a growth function, the $\lambda$-type of $F$ is defined as the number

$$
\sigma_{F}^{\lambda}:=\limsup _{|z| \rightarrow \infty} \frac{\log ^{+}|F(z)|}{\lambda(|z|)} \in[0, \infty] .
$$

If $\mathcal{H}$ is a de Branges space and $\lambda$ is a growth function, the $\lambda$-type of $\mathcal{H}$ is the number

$$
\sigma_{\mathcal{H}}^{\lambda}:=\sup _{F \in \mathcal{H}} \sigma_{F}^{\lambda} \in[0, \infty]
$$

A closed linear subspace $\mathcal{L}$ of a de Branges space $\mathcal{H}$ is called a $d B$-subspace if $\mathcal{L}$ itself, with the norm inherited from $\mathcal{H}$, is a de Branges space. The set of all dB-subspaces of a given space $\mathcal{H}$ will be denoted by $\operatorname{Sub} \mathcal{H}$. One of the most important results in the theory of de Branges spaces, the so-called Ordering Theorem, states that $\mathrm{Sub} \mathcal{H}$ is totally ordered with respect to inclusion; see [dB, Theorem 35], where an even stronger version is proved.

We address our paper to the following question: Let $\lambda$ be a growth function and let $\mathcal{H}$ be a de Branges space of finite and positive $\lambda$-type. Do there exist subspaces $\mathcal{L} \in \operatorname{Sub} \mathcal{H}$ of strictly smaller growth?

Our answer is twofold. First, we show that, given a pair $\left(\lambda, \lambda_{1}\right)$ of growth functions with

$$
\lambda_{1}(r)=o(\lambda(r)) \text { and } \lambda(r)=O(r),
$$

there exists a de Branges space $\mathcal{H}$ of finite and positive $\lambda$-type, such that one of its dBsubspaces is of finite and positive $\lambda_{1}$-type; cf. Theorem 3.6. It should be emphasized that, by [KW3, Theorem 3.10], the second condition is in a sense necessary for the existence of subspaces with smaller growth; see Remark 3.7. Second, for a de Branges space $\mathcal{H}$ with finite and positive $\lambda$-type, we give a condition ensuring that no infinite-dimensional dB-subspace can be of finite $\lambda_{1}$-type for any growth function $\lambda_{1}$ with $\lambda_{1}(r)=o(\lambda(r))$; see Theorem 4.1.

For the proof of these results we apply methods of various kinds. On the one hand, we use hard analysis, e.g., growth estimates; on the other hand, we employ more functional analytic tools, e.g., the theory of symmetric and semibounded de Branges space or the notion of transfer matrices for dB-subspaces. A cornerstone for proving the existence of examples of dB-subspaces with prescribed smaller growth is to establish the existence of de Branges spaces $\mathcal{H}$ of finite and positive $\lambda$-type (where $\lambda(r)=O(r)$ ) that have the property that the constant function 1 belongs to $\mathcal{H}+z \mathcal{H}$ (cf. Theorem 3.1), a result 
which is of interest in its own right. For the case where $\lambda(r)=r^{\rho}$, a result similar to Theorem 3.1 was proved in [BP2] by different methods.

\section{§2. Preliminaries}

In this section we recall some basic facts concerning de Branges spaces and set up some notation. Moreover, we provide a couple of results which supplement [KW3], and which will be used later on.

a. The Hermite-Biehler class. It is a basic fact that a given de Branges space $\mathcal{H}$ is completely determined by a single entire function. We say that an entire function $E$ belongs to the Hermite-Biehler class $\mathcal{H B}$ if

$$
\left|E^{\#}(z)\right|<|E(z)|, \quad z \in \mathbb{C}^{+},
$$

and $E$ has no real zeros. For $E \in \mathcal{H} \mathcal{B}$, define

$$
\mathcal{H}(E):=\left\{F \text { entire }: \frac{F}{E}, \frac{F^{\#}}{E} \in H^{2}\left(\mathbb{C}^{+}\right)\right\}
$$

where $H^{2}\left(\mathbb{C}^{+}\right)$denotes the Hardy class in the upper half-plane; see, e.g., [RosR]. Moreover, we define

$$
\|F\|_{E}^{2}:=\int_{\mathbb{R}}\left|\frac{F(t)}{E(t)}\right|^{2} d t, \quad F \in \mathcal{H}(E) .
$$

Then $\mathcal{H}(E)$ is a de Branges space. Conversely, every nonzero de Branges space can be obtained in this way. For example, the Paley-Wiener space $\mathcal{P} \mathcal{W}_{a}$ is obtained as $\mathcal{H}\left(e^{-i a z}\right)$.

By the axiom (dB1), a de Branges space $\mathcal{H}$ is a reproducing kernel Hilbert space. If $\mathcal{H}$ is a de Branges space and $E \in \mathcal{H} \mathcal{B}$ is such that $\mathcal{H}=\mathcal{H}(E)$, then the reproducing kernel $K(w, \cdot)$ of $\mathcal{H}$ can be expressed in terms of $E$ :

$$
K(w, z)=\frac{E(z) E^{\#}(\bar{w})-E(\bar{w}) E^{\#}(z)}{2 \pi i(\bar{w}-z)} .
$$

\subsection{Remark.}

(i) In the literature, the condition that $E$ has no real zeros is often dropped from the definition of $\mathcal{H B}$. This corresponds to dropping the condition $(\mathrm{Z})$ in our definition of de Branges space. As we have already remarked, dropping this condition is no essential gain in generality.

(ii) Condition (2.1) in the definition of $\mathcal{H B}$ means that the kernel $K(w, z)$ is positive semidefinite, i.e., that each of the quadratic forms $\left(z_{1}, \ldots, z_{n} \in \mathbb{C}\right)$

$$
\sum_{i, j=1}^{n} K\left(z_{j}, z_{i}\right) \zeta_{i} \overline{\zeta_{j}}
$$

is positive semidefinite. This is a classical result of analysis; see $[\mathrm{P}]$.

(iii) With an entire function $E$ we associate a pair of entire functions $A$ and $B$ :

$$
A:=\frac{E+E^{\#}}{2}, \quad B:=i \frac{E-E^{\#}}{2} .
$$

Then $A$ and $B$ are real entire functions (we say that an entire function $F$ is real if it is real on the real axis or, equivalently, if $F=F^{\#}$ ), and we have $E=A-i B$. If $E \in \mathcal{H B}$, then $A$ and $B$ have only real zeros, and these zeros are simple and interlace; that is, between every two zeros of $A$ there is a zero of $B$ and vice versa. 
(iv) The Nevanlinna class $\mathcal{N}_{0}$ is defined as the set of all functions $q$ that are analytic in $\mathbb{C} \backslash \mathbb{R}$, satisfy $q=q^{\#}$, and have nonnegative imaginary part in $\mathbb{C}^{+}$. This class of functions is closely related to $\mathcal{H B}$. More precisely, let $E$ be an entire function and write $E=A-i B$ with $A$ and $B$ as in (2.3). Then $E \in \mathcal{H B}$ if and only if $E$ has no real zeros, is not constant, and $\frac{B}{A} \in \mathcal{N}_{0}$.

\section{b. The growth of $\mathcal{H}(E)$.}

2.2. Remark. In the context of growth properties of de Branges spaces, it is a basic result that the growth of any function $F \in \mathcal{H}(E)$ is governed by the growth of $E$. In fact, we always have $\sigma_{\mathcal{H}}^{\lambda}=\sigma_{E}^{\lambda}$; see [KW3, Theorem 3.4].

We come to the announced generalizations of two results of [KW3]. They will be deduced from the next proposition, which also provides a more general viewpoint on some results concerning the exponential growth of the so-called Nevanlinna matrices; see, e.g., [BP1, Theorem 4.2], [BP2, Theorem 4.8], and [K1].

2.3. Proposition. Let $A, B$ be entire functions such that $\frac{B}{A} \in \mathcal{N}_{0}$, and let $\lambda$ be a growth function. Then $\sigma_{A}^{\lambda}=\sigma_{B}^{\lambda}$.

Proof. If both $\sigma_{A}^{\lambda}$ and $\sigma_{B}^{\lambda}$ are equal to $\infty$, we are done. We show that $\sigma_{A}^{\lambda}<\infty$ implies $\sigma_{B}^{\lambda} \leq \sigma_{A}^{\lambda}$. Since $\frac{B}{A}$ (also $-\frac{A}{B}$ ) belongs to $\mathcal{N}_{0}$, this will yield the desired conclusion.

Since the function $\frac{B}{A}$ belongs to $\mathcal{N}_{0}$, it has, by the Herglotz theorem, an integral representation of the form

$$
\frac{B(z)}{A(z)}=a z+b+\int_{\mathbb{R}}\left(\frac{1}{t-z}-\frac{t}{t^{2}+1}\right) d \mu(t), \quad z \in \mathbb{C}^{+},
$$

where $a \geq 0, b \in \mathbb{R}$, and $\mu$ is a Borel measure on $\mathbb{R}$ such that $\int_{\mathbb{R}}\left(t^{2}+1\right)^{-1} d \mu(t)<\infty$. In the present case, in fact, $\mu$ is a discrete measure with point masses at the zeros of $A$. Hence,

$$
\begin{aligned}
\left|\frac{B(z)}{A(z)}\right| & =\left|a z+b+\int_{\mathbb{R}} \frac{t z+1}{(t-z)\left(t^{2}+1\right)} d \mu(t)\right| \\
& \leq\left|a z+b+z \int_{\mathbb{R}} \frac{d \mu(t)}{t^{2}+1}\right|+\left|\left(z^{2}+1\right) \int_{\mathbb{R}} \frac{d \mu(t)}{(t-z)\left(t^{2}+1\right)}\right|, \quad z \in \mathbb{C}^{+} .
\end{aligned}
$$

Therefore,

$$
\log |B(z)| \leq \log |A(z)|+C_{1} \log (|z|+2)+\log ^{+} \frac{1}{|\operatorname{Im} z|}
$$

for all $z \in \mathbb{C} \backslash \mathbb{R}$. In particular,

$$
\log |B(z)| \leq \log |A(z)|+C_{1} \log (|z|+2), \quad|\operatorname{Im} z| \geq 1 .
$$

Now, let $|\operatorname{Im} z|<1$. Then, by the subharmonicity of $\log |B|$,

$$
\begin{aligned}
\log |B(z)| \leq & \frac{1}{2 \pi} \int_{0}^{2 \pi} \log \left|B\left(z+e^{i \phi}\right)\right| d \phi \\
\leq & \frac{1}{2 \pi} \int_{0}^{2 \pi} \log \left|A\left(z+e^{i \phi}\right)\right| d \phi+C_{2} \log (|z|+2) \\
& +\frac{1}{2 \pi} \int_{0}^{2 \pi} \log ^{+} \frac{1}{\left|\operatorname{Im}\left(z+e^{i \phi}\right)\right|} d \phi .
\end{aligned}
$$

Clearly,

$$
\frac{1}{2 \pi} \int_{0}^{2 \pi} \log ^{+} \frac{1}{\left|\operatorname{Im}\left(z+e^{i \phi}\right)\right|} d \phi \leq C_{3}
$$


for all $z$ with $|\operatorname{Im} z|<1$. Consequently,

$$
\log |B(z)| \leq \frac{1}{2 \pi} \int_{0}^{2 \pi} \log ^{+}\left|A\left(z+e^{i \phi}\right)\right| d \phi+C_{2} \log (|z|+2)+C_{3}, \quad|\operatorname{Im} z|<1 .
$$

Since $\sigma_{A}^{\lambda}<\infty$ and $\log r=o(\lambda(r))$, from (2.4) and (2.5) it follows that $\sigma_{B}^{\lambda}<\infty$ and $\sigma_{B}^{\lambda} \leq \sigma_{A}^{\lambda}$.

For a function $E \in \mathcal{H B}$, we shall always use the notation $E=A-i B$ with the real entire functions $A, B$ as in (2.3). We denote

$$
S_{\phi}:=A \sin \phi-B \cos \phi, \quad \phi \in \mathbb{R} .
$$

The family of functions $S_{\phi}$ contains significant information about the space $\mathcal{H}(E)$. In fact, the reproducing kernel $K(w, z)$ can be written in terms of the functions $S_{\phi}$. Also, the functions $S_{\phi}$ describe the selfadjoint extensions of the operator of multiplication by the independent variable in $\mathcal{H}(E)$. Clearly, $A=S_{\frac{\pi}{2}}$ and $B=S_{\pi}$. We note that

$$
S_{\phi+\frac{\pi}{2}}=\frac{1}{2}\left(e^{i \phi} E+e^{-i \phi} E^{\#}\right) .
$$

2.4. Corollary. Let $E \in \mathcal{H B}$. Then $E$ is of finite $\lambda$-type if and only if there exists one value $\phi_{0} \in \mathbb{R}$ such that $S_{\phi_{0}}$ is of finite $\lambda$-type. In this case every function $S_{\phi}, \phi \in \mathbb{R}$, is of finite $\lambda$-type, and

$$
\sigma_{E}^{\lambda}=\sigma_{S_{\phi}}^{\lambda}, \quad \phi \in \mathbb{R}
$$

Proof. It suffices to show that $\sigma_{S_{\phi}}^{\lambda}=\sigma_{E}^{\lambda}$ for any $\phi \in \mathbb{R}$. Since the functions $E(z)$ and $e^{i\left(\phi-\frac{\pi}{2}\right)} E(z)$ have the same $\lambda$-type, we can restrict the explicit proof to the case where $\phi=\frac{\pi}{2}$, i.e., $S_{\phi}=A$.

Assume that $\sigma_{A}^{\lambda}<\infty$. Then, by the definition of $A$ and $B$ and by Proposition 2.3,

$$
\sigma_{E}^{\lambda} \leq \max \left\{\sigma_{A}^{\lambda}, \sigma_{B}^{\lambda}\right\}=\sigma_{A}^{\lambda} \leq \sigma_{E}^{\lambda} .
$$

It follows that $\sigma_{E}^{\lambda}<\infty$ and, in fact, $\sigma_{E}^{\lambda}$ is equal to $\sigma_{A}^{\lambda}$. Conversely, if $\sigma_{E}^{\lambda}<\infty$, then, clearly, $\sigma_{A}^{\lambda} \leq \sigma_{E}^{\lambda}$ and, thus, $\sigma_{A}^{\lambda}<\infty$.

We obtain a generalization of [KW3, Corollary 3.18], where the following statement was proved under the additional assumption $r=O(\lambda(r))$.

2.5. Corollary. Let $\mathcal{H}$ be a de Branges space, and let $\lambda$ be a growth function. Then $\mathcal{H}$ is of finite $\lambda$-type if and only if for some $\phi \in[0, \pi)$ the function $S_{\phi}$ is of finite $\lambda$-type. In this case $\sigma_{\mathcal{H}}^{\lambda}=\sigma_{S_{\phi}}^{\lambda}$.

Proof. Combine Corollary 2.4 with Remark 2.2.

We also obtain a similar generalization of [KW3, Theorem 3.17], i.e., we can drop the assumption $r=O(\lambda(r))$ made there. In order to explain this result, we need to recall the notion of a $\lambda$-admissible sequence of complex numbers; $\mathrm{cf}$. $[\mathrm{Ru}]$.

We say that a sequence $\left\{z_{n}\right\}$ has a finite $\lambda$-density if

$$
\sum_{\left|z_{n}\right| \leq r} \log \frac{r}{\left|z_{n}\right|}=O(\lambda(r)) .
$$

When comparing with the definition in $[\mathrm{Ru}]$ one should be aware of the property $\lambda(r) \asymp$ $\lambda(c r)$ for any $c>1$, which follows from the definition of a growth function (we write $f \asymp g$ if there exist constants $c_{1}, c_{2}>0$ such that $c_{1} f(x) \leq g(x) \leq c_{2} f(x)$ for all admissible values of $x$ ). The sequence $\left\{z_{n}\right\}$ is said to be $\lambda$-balanced if, uniformly in $k \in \mathbb{N}$, we have

$$
\left|\frac{1}{k} \sum_{r_{1}<\left|z_{n}\right| \leq r_{2}}\left(\frac{1}{z_{n}}\right)^{k}\right|=O\left(\frac{\lambda\left(r_{1}\right)}{r_{1}^{k}}+\frac{\lambda\left(r_{2}\right)}{r_{2}^{k}}\right), \quad r_{1}, r_{2} \rightarrow \infty .
$$


Finally, the sequence $\left\{z_{n}\right\}$ is $\lambda$-admissible if it is both of finite $\lambda$-density and $\lambda$-balanced.

2.6. Corollary. $A$ de Branges space $\mathcal{H}(E)$ is obtained from a de Branges space $\mathcal{H}\left(E_{1}\right)$ of finite $\lambda$-type by multiplication by a zero free entire function real on $\mathbb{R}$ if and only if for one (and, hence, for all) $\phi \in[0, \pi)$ the sequence of zeros of $S_{\phi}$ is $\lambda$-admissible.

Proof. We repeat briefly the proof in [KW3]. If the sequence $\left\{a_{n}\right\}$ of zeros of $S_{\phi}$ is $\lambda$-admissible, then, by [Ru, Theorem 13.5.2], there exists an entire function $A_{1}$ of finite $\lambda$-type, $A_{1}=A_{1}^{\#}$, having $\left\{a_{n}\right\}$ as its precise set of zeros. Hence, $C=\frac{S_{\phi}}{A_{1}}$ is a zero free entire function real on $\mathbb{R}$. Put

$$
E_{1}(z)=\frac{S_{\phi}(z)-i S_{\phi+\frac{\pi}{2}}(z)}{C(z)}=-\frac{i e^{i \phi} E(z)}{C(z)} .
$$

Then $E_{1} \in \mathcal{H B}$ and $\mathcal{H}(E)=\mathcal{H}\left(C E_{1}\right)=C \mathcal{H}\left(E_{1}\right)$. Note also that $\frac{E_{1}+E_{1}^{\#}}{2}=A_{1}$ is of finite $\lambda$-type. By Corollary 2.4, $E_{1}$ is of finite $\lambda$-type and, thus, $\mathcal{H}\left(E_{1}\right)$ is of finite $\lambda$-type.

The converse implication is almost immediate; see [KW3] for details.

c. Functions associated with a de Branges space. An entire function $S$ is said to be associated with the space $\mathcal{H}(E)$ if for each $w \in \mathbb{C}$ the difference quotient operator

$$
F(z) \mapsto \frac{F(z) S(w)-S(z) F(w)}{z-w}
$$

maps the space $\mathcal{H}(E)$ into itself. The set of all functions associated with a space $\mathcal{H}(E)$ is denoted by $\operatorname{Assoc} \mathcal{H}(E)$.

The functions of class Assoc $\mathcal{H}(E)$ can be characterized in various ways. For instance, it is straightforward to verify that

$$
\operatorname{Assoc} \mathcal{H}(E)=\mathcal{H}(E)+z \mathcal{H}(E) .
$$

Another characterization of Assoc $\mathcal{H}(E)$ employs a certain class of entire matrix functions. This is a deep result; see [dB, Theorem 27].

The spaces $\mathcal{H}(E)$ with the property that $1 \in \operatorname{Assoc} \mathcal{H}(E)$ are of particular interest. Let us formulate explicitly the characterization [dB, Theorem 27] (mentioned above) for this case. For this, we need to recall the notion of matrix functions of class $\mathcal{M}_{0}$, a notion closely related to the so-called Nevanlinna matrices. A $(2 \times 2)$-matrix $W=\left(w_{i j}\right)_{i, j=1,2}$ is said to belong to the class $\mathcal{M}_{0}$ if its entries $w_{i j}$ are real entire functions, $W(0)=I$, $\operatorname{det} W \equiv 1$, and the kernel

$$
H_{W}(w, z):=\frac{W(z) J W^{*}(w)-J}{z-\bar{w}}
$$

where

$$
J:=\left(\begin{array}{cc}
0 & -1 \\
1 & 0
\end{array}\right)
$$

is positive semidefinite. We note that if

$$
W=\left(\begin{array}{ll}
A_{W} & B_{W} \\
C_{W} & D_{W}
\end{array}\right) \in \mathcal{M}_{0}
$$

then each of the functions $A_{W}-i B_{W}, D_{W}+i C_{W}, D_{W}-i B_{W}$, and $A_{W}+i C_{W}$ belongs to $\mathcal{H B}$.

The relationship between $\mathcal{M}_{0}$ and the spaces $\mathcal{H}(E)$ with the property that $1 \in$ $\operatorname{Assoc} \mathcal{H}(E)$ is the following. 
2.7. Remark. Let $W \in \mathcal{M}_{0}$ and define

$$
E_{W}:=A_{W}-i B_{W}, \quad\left(A_{W}, B_{W}\right):=(1,0) W .
$$

Then $E_{W} \in \mathcal{H B}$ and $1 \in \operatorname{Assoc} \mathcal{H}\left(E_{W}\right)$. Conversely, if $\mathcal{H}$ is a de Branges space with $1 \in \operatorname{Assoc} \mathcal{H}$, and we write $\mathcal{H}=\mathcal{H}(E), E=A-i B$, with $E(0)=1$, then there exist entire functions $C, D$ such that

$$
W:=\left(\begin{array}{cc}
A & B \\
C & D
\end{array}\right) \in \mathcal{M}_{0}
$$

In this case the functions $D, C$ can be chosen so that

$$
\lim _{y \rightarrow+\infty} \frac{1}{y} \operatorname{Im} \frac{D(i y)}{B(i y)}=0 .
$$

We shall also employ the following result, which was proved in [W, Theorem 1.1], and from which a characterization of $1 \in \operatorname{Assoc} \mathcal{H}(E)$ can be deduced.

2.8. Remark. Let $E=A-i B \in \mathcal{H B}$ and $E(0)=1$. Then the set Assoc $\mathcal{H}(E)$ contains a real and zero free function if and only if the following conditions are satisfied:

(C1) Let $\left(x_{k}\right)_{k \in \mathbb{N}}$ be the sequence of zeros of $A$. Then the $\operatorname{limit} \lim _{r \rightarrow \infty} \sum_{\left|a_{k}\right| \leq r} \frac{1}{x_{k}}$ exists in $\mathbb{R}$.

(C2) Let $\left(x_{k}^{+}\right)_{k \in \mathbb{N}}$ and $\left(x_{k}^{-}\right)_{k \in \mathbb{N}}$ be the sequence of positive and negative, respectively, zeros of $A$ arranged according to increasing modulus. Then the limits $\lim _{k \rightarrow \infty} \frac{k}{x_{k}^{+}}$ and $\lim _{k \rightarrow \infty} \frac{k}{x_{k}^{-}}$exist in $\mathbb{R}$ and are equal.

(C3) Let $X(z):=\lim _{r \rightarrow \infty} \prod_{\left|x_{k}\right| \leq r}\left(1-\frac{z}{x_{k}}\right)$ and $Y(z):=z \lim _{r \rightarrow \infty} \prod_{\left|y_{k}\right| \leq r}\left(1-\frac{z}{y_{k}}\right)$, where $\left(y_{k}\right)_{k \in \mathbb{N}}$ denotes the sequence of nonzero zeros of $B$. Then

$$
\sum_{k \in \mathbb{N}}\left|\frac{1}{x_{k}^{2} X^{\prime}\left(x_{k}\right) Y\left(x_{k}\right)}\right|<\infty .
$$

In this case we have $\frac{A(z)}{X(z)} \in \operatorname{Assoc} \mathcal{H}(E)$.

The class $\mathcal{M}_{0}$ plays also in other respects an important role in the theory of de Branges spaces; cf. [dB, Theorems 33, 34].

2.9. Remark. If $W$ and $W_{1}$ belong to $\mathcal{M}_{0}$, then $W W_{1} \in \mathcal{M}_{0}$. We have $\mathcal{H}\left(E_{W}\right) \subseteq$ $\mathcal{H}\left(E_{W W_{1}}\right)$, and the set-theoretic inclusion map is a contraction. If the set $\mathcal{H}\left(E_{W}\right)$ is endowed with the norm $\|\cdot\|_{E_{W W_{1}}}$, then it becomes a dB-subspace of $\mathcal{H}\left(E_{W W_{1}}\right)$.

d. Transformations of matrices of class $\mathcal{M}_{0}$. We shall use some transformations of matrices.

2.10. Definition. Let $W$ be a $(2 \times 2)$-matrix function whose elements are entire functions.

(i) We put

$$
V:=\left(\begin{array}{ll}
0 & 1 \\
1 & 0
\end{array}\right)
$$

and define

$$
\mathfrak{v}(W):=V W^{-1} V .
$$

(ii) For $a \in \mathbb{R}$, we define

$$
\mathfrak{w}_{a}(W)(z):=W(a)^{-1} W(z+a) .
$$


Then, as a computation of reproducing kernels shows, for $a \in \mathbb{R}$ the maps $\mathfrak{v}$ and $\mathfrak{w}_{a}$ take $\mathcal{M}_{0}$ into itself; see, e.g., [KWW1, Lemma 2.3] and [Wi].

If $\lambda$ is a growth function and $W=\left(w_{i j}\right)_{i, j=1,2} \in \mathcal{M}_{0}$, then, by Proposition 2.3, all the entries $w_{i j}$ of $W$ are of the same $\lambda$-type. This allows us to put

$$
\sigma_{W}^{\lambda}:=\sigma_{w_{i j}}^{\lambda}, \quad i, j=1,2 .
$$

2.11. Lemma. Let $W \in \mathcal{M}_{0}$. Then $\sigma_{\mathcal{H}\left(E_{W}\right)}^{\lambda}=\sigma_{W}^{\lambda}$. We have

$$
\sigma_{\mathfrak{v}(W)}^{\lambda}=\sigma_{\mathfrak{w}_{a}(W)}^{\lambda}=\sigma_{W}^{\lambda}, \quad a \in \mathbb{R} .
$$

If, moreover, $W_{1} \in \mathcal{M}_{0}$, then

$$
\sigma_{W}^{\lambda} \leq \sigma_{W W_{1}}^{\lambda} \leq \sigma_{W}^{\lambda}+\sigma_{W_{1}}^{\lambda}
$$

Proof. The first assertion follows from Corollary 2.4. The relation $\sigma_{\mathfrak{v}(W)}^{\lambda}=\sigma_{W}^{\lambda}$ is immediate from the explicit computation

$$
\mathfrak{v}(W)=\left(\begin{array}{cc}
w_{11} & -w_{21} \\
-w_{12} & w_{22}
\end{array}\right)
$$

The fact that $\sigma_{\mathfrak{w}_{a}(W)}^{\lambda}=\sigma_{W}^{\lambda}$ follows because $W(a)$ is invertible and because for any entire function $f$ we have $\sigma_{f(z)}^{\lambda}=\sigma_{f(z+a)}^{\lambda}$.

The first inequality in (2.10) is a consequence of the fact that, as a set, $\mathcal{H}\left(E_{W}\right)$ is contained in $\mathcal{H}\left(E_{W W_{1}}\right)$. The second inequality in (2.10) is trivial.

e. The square root transformation. The following transformation of entire functions is a useful tool. Let $E$ be an entire function and assume that $E$ satisfies the functional equation

$$
E^{\#}(z)=E(-z)
$$

If $E$ is written as $E=A-i B$ with $A$ and $B$ as in (2.3), we define $E_{+}:=A_{+}-i B_{+}$, where $A_{+}$and $B_{+}$are defined by the relations

$$
A_{+}\left(z^{2}\right)=A(z), \quad B_{+}\left(z^{2}\right)=z B(z) .
$$

Note that the validity of $(2.11)$ is equivalent to $A$ being even and $B$ being odd, so that $A_{+}$and $B_{+}$are well-defined entire functions. Clearly, $B_{+}(0)=0$.

The assignment $E \mapsto E_{+}$maps the set of all entire functions satisfying (2.11) bijectively onto the set of all entire functions that take a real value at 0 . Its inverse is given by

$$
E_{+}=A_{+}-i B_{+} \mapsto E:=A_{+}\left(z^{2}\right)-\frac{i}{z} B_{+}\left(z^{2}\right) .
$$

In connection with this transformation, the so-called Stieltjes class $\mathcal{S}$ is of importance. Recall that a function $q$ is said to belong to $\mathcal{S}$ if $q \in \mathcal{N}_{0}$ and is analytic and nonnegative on $(-\infty, 0)$. The fact that $q \in \mathcal{S}$ can be characterized in several ways; see [KaK1, KaK2]. We recall that

$$
q \in \mathcal{S} \Longleftrightarrow z q\left(z^{2}\right) \in \mathcal{N}_{0} .
$$

Moreover, for any function $q \in \mathcal{S}$ we have $\lim _{y \rightarrow+\infty} \frac{1}{y} \operatorname{Im} q(i y)=0$ and $\lim _{x \rightarrow-\infty} q(x) \in$ $\mathbb{R}$.

2.12. Remark. Let $A-i B \in \mathcal{H B}$, and assume that $A$ and $B$ do not vanish on $(-\infty, 0)$ while $B(0)=0$. Then $q=-\frac{A}{B} \in \mathcal{S}$. Indeed, $q \in \mathcal{N}_{0}$, and therefore, by the Herglotz theorem,

$$
q(z)=-\frac{\sigma_{0}}{z}+\sum_{n=1}^{\infty} \sigma_{n}\left(\frac{1}{x_{n}-z}-\frac{1}{x_{n}}\right)+a+b z
$$


for some $a \in \mathbb{R}, b \geq 0$, and $\sigma_{n}>0, n=0,1, \ldots$. Here the $x_{n}$ are the nonzero zeros of $B$. Clearly, $\lim _{x \rightarrow-0} q(x)=+\infty$. Since $q$ does not change sign on $(-\infty, 0)$, it follows that $q>0$ on $(-\infty, 0)$.

2.13. Remark. Let $E=A-i B$ and $E_{+}=A_{+}-i B_{+}$be entire functions related to each other as in (2.12). Then:

(i) $E \in \mathcal{H B}$ if and only if $E_{+} \in \mathcal{H B}$ and $A_{+}(x) B_{+}(x)<0, x<0$, which is equivalent to $-\frac{A_{+}}{B_{+}} \in \mathcal{S}$. This follows by combining Remark 2.1, (iv), with (2.13) and the fact that $q \in \mathcal{N}_{0}$ if and only if $-\frac{1}{q} \in \mathcal{N}_{0}$.

(ii) If $E \in \mathcal{H B}$ and $1 \in \operatorname{Assoc} \mathcal{H}(E)$, then also $1 \in \operatorname{Assoc} \mathcal{H}\left(E_{+}\right)$. This follows from some geometric arguments concerning the generated de Branges spaces. A proof can be found in [KWW2, Proposition 2.6], where even a more general setting was considered.

(iii) Assume that $E \in \mathcal{H B}$ and $1 \in \operatorname{Assoc} \mathcal{H}\left(E_{+}\right)$. Let $C_{+}, D_{+}$be entire functions as in Remark 2.7 and satisfying (2.8). Then $1 \in \operatorname{Assoc} \mathcal{H}(E)$ if and only if

$$
\lim _{x \rightarrow-\infty} \frac{D_{+}(x)}{B_{+}(x)} \in \mathbb{R} .
$$

At first sight, this result may look surprising. It follows from a deeper discussion concerning the reproducing kernel space generated by the matrix $W$ in (2.7) (see [KWW1, Proposition 3.14]), which involves the theory of de Branges Pontryagin spaces; cf. [KW1]. No direct proof is known to the authors.

\section{§3. Existence of SUbSPACES WITH PRESCRIBED GROWTH}

This section is devoted to the proof of the existence of de Branges spaces that have subspaces of smaller growth; see Theorem 3.6. Our method is based on the following result, which is also of interest in its own right.

3.1. Theorem. Let $\lambda$ be a growth function with $\lambda(r)=O(r)$. Then there exists a de Branges space $\mathcal{H}$ of finite and positive $\lambda$-type with $1 \in \operatorname{Assoc} \mathcal{H}$.

Before proceeding to the proof of this theorem, we discuss the statement in more detail.

\subsection{Remark.}

(i) By [KW3, Theorem 3.10], every de Branges space $\mathcal{H}$ with $1 \in$ Assoc $\mathcal{H}$ must be of finite exponential type. Hence, the condition $\lambda(r)=O(r)$ is natural.

(ii) A different approach to this theorem could proceed via [BP2, Theorems 3.6, 5.1]; the method employed there is function-theoretic in its nature and goes back to a result of Krein; see [K2]. By means of this approach the case where $\lambda(r)=r^{\rho}$ was treated in [BP2, Theorems 5.6]. In the present paper, however, we prefer a more Hilbert-space-theoretic point of view.

(iii) The hard part of the theorem is to deal with the case where $\lambda(r)$ grows more slowly, but almost as quickly as $r$. If the growth function $\lambda, \lambda(r)=O(r)$, satisfies a very mild additional condition, a space $\mathcal{H}$ with finite and positive $\lambda$-type and with $1 \in \operatorname{Assoc} \mathcal{H}$ can be constructed much more explicitly. Indeed, denoting by $\mu$ the inverse function of $\lambda$, let us assume that

$$
\int_{0}^{\infty} \frac{d t}{\mu(t)}<\infty
$$


Put $y_{n}:=\mu(n), n \in \mathbb{N}$. Then, by (3.1), we have $\sum_{n=1}^{\infty} \frac{1}{y_{n}}<\infty$. Hence, we may define an entire function by

$$
E(z):=\prod_{n \in \mathbb{N}}\left(1+\frac{z}{i y_{n}}\right)
$$

Clearly, $E \in \mathcal{H} \mathcal{B}$. Moreover, e.g., by [B2, Theorem 1], the space $\mathcal{H}(E)$ contains the set of all polynomials as a dense linear subspace. In particular, $1 \in \operatorname{Assoc} \mathcal{H}(E)$. Since

$$
\Delta_{\lambda}\left(\left(y_{n}\right)_{n \in \mathbb{N}}\right)=1, \quad \limsup _{r \rightarrow \infty}\left|\sum_{\left|i y_{n}\right| \leq r} \frac{1}{i y_{n}}\right|=\sum_{n=1}^{\infty} \frac{1}{y_{n}}<\infty,
$$

the results of $[\mathrm{L}, \mathrm{I}$. Lehrsatz 17,18$]$ imply that $E$ is of finite and positive $\lambda$-type.

Note that if (3.1) fails, a similar construction is not possible. This follows from the fact that, due to the presence of the Blaschke condition, a function of finite and positive $\lambda$-type must, in the case where (3.1) fails, have most of its zeros close to the real axis in the sense that there exists no angle $\{z \in \mathbb{C}: \arg z \in(-\pi+\delta,-\delta)\}$, $\delta>0$, containing all the zeros of $E$.

Let us come to the proof of Theorem 3.1. It is based on a perturbation argument. Let $E=A-i B \in \mathcal{H B}, E(0)=1$, and let $\left(\gamma_{k}\right)_{k \in \mathbb{N}}$ be a monotone nondecreasing sequence of positive real numbers. Assume the following:

(a) All zeros of $A$ lie in $(0, \infty)$.

(b) Denote by $\left(a_{k}\right)_{k \in \mathbb{N}}$ the sequence of zeros of $A$ arranged increasingly. Then

$$
\sum_{k=1}^{\infty} \frac{1}{a_{k}}<\infty
$$

Note that $B(0)=0$ because $E(0)=1$. Taking into account (a) and the fact that the zeros of $A$ and $B$ interchange, we see that the zeros of $B$ lie in $[0, \infty)$.

We construct a function $\tilde{E}(z)$ by proceeding as follows.

(1) The function $\frac{B(z)}{A(z)}$ belongs to the class $\mathcal{N}_{0}$ and is meromorphic in $\mathbb{C}$. Hence, if we put $\sigma_{n}:=-\frac{B\left(a_{n}\right)}{A^{\prime}\left(a_{n}\right)}$, then $\sigma_{n}>0, \sum_{k=1}^{\infty} \sigma_{k} a_{k}^{-2}<\infty$, and we have

$$
\frac{B(z)}{A(z)}=a z+\sum_{k=1}^{\infty}\left(\frac{1}{a_{k}-z}-\frac{1}{a_{k}}\right) \sigma_{k}
$$

where $a \geq 0$.

(2) Define a sequence $\left(\tilde{a}_{k}\right)_{k \in \mathbb{N}}$ as $\tilde{a}_{k}:=a_{k} \gamma_{k}, k \in \mathbb{N}$. Then

$$
0<\tilde{a}_{1}<\tilde{a}_{2}<\cdots \quad \text { and } \quad \tilde{a}_{k} \geq \gamma_{1} a_{k}, \quad k \in \mathbb{N} .
$$

(3) We have

$$
\sum_{k=1}^{\infty} \frac{\sigma_{k}}{1+\tilde{a}_{k}^{2}} \leq \sum_{k=1}^{\infty} \frac{\sigma_{k}}{1+\gamma_{1}^{2} a_{k}^{2}}<\infty .
$$

Consequently, the series

$$
\tilde{q}(z):=\sum_{k=1}^{\infty}\left(\frac{1}{\tilde{a}_{k}-z}-\frac{1}{\tilde{a}_{k}}\right) \sigma_{k}
$$

determines a function $\tilde{q} \in \mathcal{N}_{0}$ meromorphic in $\mathbb{C}$ and such that its poles are the $\tilde{a}_{k}$, and its residue at $\tilde{a}_{k}$ is $-\sigma_{k} \neq 0$. 
(4) We have

so that the product

$$
\sum_{k=1}^{\infty} \frac{1}{\tilde{a}_{k}} \leq \sum_{k=1}^{\infty} \frac{1}{\gamma_{1} a_{k}}<\infty
$$

$$
\tilde{A}(z):=\prod_{k=1}^{\infty}\left(1-\frac{z}{\tilde{a}_{k}}\right)
$$

determines an entire function.

(5) Put $\tilde{B}(z):=\tilde{q}(z) \cdot \tilde{A}(z)$; then $\tilde{B}$ is a real entire function and $\tilde{B}(0)=0$. If we now define

then $\tilde{E} \in \mathcal{H B}$ (see Remark 2.1(iv)).

$$
\tilde{E}:=\tilde{A}-i \tilde{B},
$$

3.3. Lemma. Assume that $E \in \mathcal{H B}$ is subject to the above conditions (a) and (b), and let $\tilde{E}$ be constructed as in (1)-(5). If $1 \in \operatorname{Assoc} \mathcal{H}(E)$, then also $1 \in \operatorname{Assoc} \mathcal{H}(\tilde{E})$.

Proof. Assume that $1 \in \operatorname{Assoc} \mathcal{H}(E)$. In order to prove the lemma, we shall verify the conditions $(\mathrm{C} 1)-(\mathrm{C} 3)$ of Remark 2.8 for the function $\tilde{E}=\tilde{A}-i \tilde{B}$.

Because of the positivity of the numbers $\tilde{a}_{k}$, condition $(\mathrm{C} 1)$ is equivalent to the convergence of the series $\sum_{k \in \mathbb{N}} \frac{1}{\tilde{a}_{k}}$, and this was already seen in item (4) above. Condition (C2) is fulfilled because $\left(\gamma_{k}\right)_{k \in \mathbb{N}}$ is monotone increasing (thus, $\lim _{k \rightarrow \infty} \frac{1}{\gamma_{k}}$ exists), and because, by the assumption of the lemma, the sequence $\left(a_{k}\right)_{k \in \mathbb{N}}$ satisfies (C2). Indeed, since all zeros are positive, $\lim _{k \rightarrow \infty} \frac{k}{a_{k}}=0$ and hence also $\lim _{k \rightarrow \infty} \frac{k}{\tilde{a}_{k}}=0$.

To establish (C3), we need to estimate $\tilde{A}^{\prime}\left(\tilde{a}_{k}\right)$. Since $1 \in \operatorname{Assoc} \mathcal{H}(E)$ and all zeros of $A$ are positive, [KWW1, Proposition 3.12] implies that $E$, and hence also $A$ and $B$, are of order at most $\frac{1}{2}$. Thus, $A(z)=\prod_{n=1}^{\infty}\left(1-\frac{z}{a_{n}}\right)$ and $B(z)=\alpha z \prod_{n=1}^{\infty}\left(1-\frac{z}{b_{n}}\right)$, where $\left(b_{n}\right)_{n \in \mathbb{N}}$ denotes the sequence of nonzero zeros of $B$, and $\alpha>0$. Note that, by locally uniform convergence of the respective products and analyticity,

$$
\tilde{A}^{\prime}\left(\tilde{a}_{k}\right)=-\frac{1}{\tilde{a}_{k}} \prod_{\substack{n \in \mathbb{N} \\ n \neq k}}\left(1-\frac{\tilde{a}_{k}}{\tilde{a}_{n}}\right), \quad A^{\prime}\left(a_{k}\right)=-\frac{1}{a_{k}} \prod_{\substack{n \in \mathbb{N} \\ n \neq k}}\left(1-\frac{a_{k}}{a_{n}}\right) .
$$

We have $\frac{\tilde{a}_{k}}{\tilde{a}_{n}}=\frac{a_{k}}{a_{n}} \frac{\gamma_{k}}{\gamma_{n}}$, whence

$$
\frac{\tilde{a}_{k}}{\tilde{a}_{n}} \begin{cases}\leq \frac{a_{k}}{a_{n}} & \text { if } k<n, \\ \geq \frac{a_{k}}{a_{n}} & \text { if } k>n\end{cases}
$$

Moreover,

$$
\left|1-\frac{\tilde{a}_{k}}{\tilde{a}_{n}}\right|=\left\{\begin{array}{ll}
1-\frac{\tilde{a}_{k}}{\tilde{a}_{n}} & \text { if } k<n \\
\frac{\tilde{a}_{k}}{\tilde{a}_{n}}-1 & \text { if } k>n
\end{array}\right\} \geq\left\{\begin{array}{ll}
1-\frac{a_{k}}{a_{n}} & \text { if } k<n \\
\frac{a_{k}}{a_{n}}-1 & \text { if } k>n
\end{array}\right\}=\left|1-\frac{a_{k}}{a_{n}}\right| .
$$

It follows that

$$
\left|\tilde{A}^{\prime}\left(\tilde{a}_{k}\right)\right| \geq \frac{1}{\gamma_{k}}\left|A^{\prime}\left(a_{k}\right)\right| .
$$

The series (2.9) for $E$ can be written as

$$
\sum_{k \in \mathbb{N}}\left|\frac{1}{a_{k}^{2} A^{\prime}\left(a_{k}\right) B\left(a_{k}\right)}\right|=\sum_{k \in \mathbb{N}} \frac{1}{a_{k}^{2} A^{\prime}\left(a_{k}\right)^{2} \sigma_{k}},
$$

and, since by our construction the numbers $\sigma_{k}$ are the same for $E$ and $\tilde{E}$, the series (2.9) for $\tilde{E}$ is nothing else but

$$
\sum_{k \in \mathbb{N}} \frac{1}{\tilde{a}_{k}^{2} \tilde{A}^{\prime}\left(\tilde{a}_{k}\right)^{2} \sigma_{k}} .
$$


It follows that

$$
\sum_{k \in \mathbb{N}} \frac{1}{\tilde{a}_{k}^{2} \tilde{A}^{\prime}\left(\tilde{a}_{k}\right)^{2} \sigma_{k}} \leq \sum_{k \in \mathbb{N}} \frac{1}{\left(\gamma_{k} a_{k}\right)^{2}\left(\frac{1}{\gamma_{k}} A^{\prime}\left(a_{k}\right)\right)^{2} \sigma_{k}}=\sum_{k \in \mathbb{N}} \frac{1}{a_{k}^{2} A^{\prime}\left(a_{k}\right)^{2} \sigma_{k}}<\infty,
$$

i.e., condition (C3) is satisfied.

The next lemma is completely elementary. It simply ensures that the numbers $\gamma_{n}$ can be chosen appropriately. Recall that for a growth function $\mu$ the upper $\mu$-density of a sequence $\left(w_{k}\right)_{k \in \mathbb{N}}$ of complex numbers is defined as

$$
\Delta_{\mu}\left(\left(w_{k}\right)_{k \in \mathbb{N}}\right):=\limsup _{r \rightarrow \infty} \frac{\#\left\{k \in \mathbb{N}:\left|w_{k}\right| \leq r\right\}}{\mu(r)} .
$$

3.4. Lemma. Let $\lambda$ and $\lambda_{1}$ be growth functions with $\lambda_{1}(r)=O(\lambda(r))$, and let $\left(a_{k}\right)_{k \in \mathbb{N}}$ be a monotone increasing sequence of positive real numbers that has a finite and positive upper $\lambda$-density. Then there exists a monotone nondecreasing sequence $\left(\gamma_{k}\right)_{k \in \mathbb{N}}$ of positive real numbers such that the sequence $\left(\gamma_{k} a_{k}\right)_{k \in \mathbb{N}}$ has a finite and positive upper $\lambda_{1}$-density.

Proof. Without loss of generality we can assume that $\lambda_{1}$ is continuous and monotone increasing, and that $\lim _{r \searrow 0} \lambda_{1}(r) \in(0,1)$.

First, observe that, since $\lambda_{1}(r) \leq c \lambda(r)$ for an appropriate real constant $c$, we certainly have

$$
\Delta_{\lambda_{1}}\left(\left(a_{k}\right)_{k \in \mathbb{N}}\right) \geq \frac{1}{c} \Delta_{\lambda}\left(\left(a_{k}\right)_{k \in \mathbb{N}}\right)>0 .
$$

If $\Delta_{\lambda_{1}}\left(\left(a_{k}\right)_{k \in \mathbb{N}}\right)<\infty$, then we set $\gamma_{n}:=1, n \in \mathbb{N}$, and we are done. Hence, for the rest of the proof we may assume that $\Delta_{\lambda_{1}}\left(\left(a_{k}\right)_{k \in \mathbb{N}}\right)=\infty$.

Inductively, we construct numbers $n_{k} \in \mathbb{N}$ and real numbers $\gamma_{1}, \ldots, \gamma_{n_{k}}$ such that

(i) $n_{k}<n_{k+1}, k \in \mathbb{N}$;

(ii) $0<\gamma_{1} \leq \cdots \leq \gamma_{n_{k}}$;

(iii) for all $0<r \leq \gamma_{n_{k}} a_{n_{k}}$ we have

$$
\frac{\#\left\{n: \gamma_{n} a_{n} \leq r\right\}}{\lambda_{1}(r)} \leq 1
$$

Moreover, the construction will be carried out in such a way that

(iv) for all $k>1$ there exists $r_{k}>0$ such that $r_{k} \rightarrow \infty$ and

$$
\lim _{k \rightarrow \infty} \frac{\#\left\{\gamma_{n} a_{n} \leq r_{k}\right\}}{\lambda_{1}\left(r_{k}\right)}=1 .
$$

For $k=1$ we put $n_{1}:=1$, and let $\gamma_{1}$ be such that $\lambda_{1}\left(\gamma_{1} a_{1}\right)=1$. Then (i) and (ii) are trivial, and (iii) is fulfilled because $\left\{\gamma_{n} a_{n} \leq r\right\}=\varnothing$ for $r<\gamma_{1} a_{1}$.

We come to the inductive step. Assume that numbers $n_{k}$ and $\gamma_{l}$ satisfying (i)-(iii) are given for all $k \leq K$ and $l=1, \ldots, n_{K}$. Consider the sequence

$$
\tilde{a}_{n}:= \begin{cases}\gamma_{n} a_{n} & \text { if } n \leq n_{K}, \\ \gamma_{n_{K}} a_{n} & \text { if } n>n_{K} .\end{cases}
$$

Consider the function $g(r):=\frac{\#\left\{\tilde{a}_{n} \leq r\right\}}{\lambda_{1}(r)}$. It is continuous from the right, has positive jumps at the points $\tilde{a}_{n}$, and is monotone decreasing between two successive jumps. By (iii) of the inductive hypothesis,

$$
g\left(\tilde{a}_{n_{K}}\right)=\frac{\#\left\{n: \gamma_{n} a_{n} \leq \gamma_{n_{K}} a_{n_{K}}\right\}}{\lambda_{1}\left(\gamma_{n_{K}} a_{n_{K}}\right)} \leq 1
$$


Moreover, since $\lambda_{1}\left(\gamma_{n_{K}} r\right) \asymp \lambda_{1}(r)$ for any fixed $K$, we have $\Delta_{\lambda_{1}}\left(\left(\tilde{a}_{n}\right)_{n \in \mathbb{N}}\right)=\infty$, i.e., $\limsup _{r \rightarrow \infty} g(r)=\infty$. Hence, there exists a smallest number $r$ that satisfies

$$
r>\tilde{a}_{n_{K}} \text { and } g(r) \geq 1
$$

Clearly, we must have $r=\tilde{a}_{n}$ for some $n \in \mathbb{N}$. We define $n_{K+1}$ as this number $n$. Then $n_{K+1}>n_{K}$, and (i) is satisfied. Consider the function

$$
f(\alpha):=\frac{n_{K}+\#\left\{n>n_{K}: \alpha \tilde{a}_{n} \leq \tilde{a}_{n_{K+1}}\right\}}{\lambda_{1}\left(\tilde{a}_{n_{K+1}}\right)} .
$$

This is a monotone nonincreasing step function with jumps of height $\lambda_{1}\left(\tilde{a}_{n_{K+1}}\right)^{-1}$. We have $f(1)=g\left(\tilde{a}_{n_{K+1}}\right) \geq 1$, and

$$
f(\alpha)=\frac{n_{K}}{\lambda_{1}\left(\tilde{a}_{n_{K+1}}\right)} \leq \frac{n_{K}}{\lambda_{1}\left(\tilde{a}_{n_{K}}\right)} \leq 1
$$

whenever $\alpha>\tilde{a}_{n_{K+1}} \tilde{a}_{n_{K}+1}^{-1}$. Thus, there exists a value $\alpha_{0} \in[1, \infty)$ such that $1 \geq f\left(\alpha_{0}\right) \geq$ $1-\lambda_{1}\left(\tilde{a}_{n_{K+1}}\right)^{-1}$. We define

$$
\gamma_{l}:=\alpha_{0} \gamma_{n_{K}}, \quad n_{K}<l \leq n_{K+1} .
$$

The requirement (iii) for the values $r \leq \gamma_{n_{K}}$ is fulfilled by the inductive hypothesis. For $\gamma_{n_{K}}<r \leq \gamma_{n_{K+1}}$, this requirement follows from our choice of $n_{K+1}$ and the fact that $\alpha_{0} \geq 1$.

Finally, put $r_{k}=\lambda_{1}\left(\tilde{a}_{n_{K+1}}\right)=\lambda_{1}\left(\gamma_{n_{K}} a_{n_{K+1}}\right)$. Then, by our choice of $\alpha_{0}$,

$$
\frac{\#\left\{\gamma_{n} a_{n} \leq r_{k}\right\}}{\lambda_{1}\left(r_{k}\right)}=f\left(\alpha_{0}\right) \geq 1-\lambda_{1}\left(\tilde{a}_{n_{K+1}}\right)^{-1}
$$

and condition (iv) is satisfied.

Proof of Theorem 3.1. Consider the Paley-Wiener space $\mathcal{P} \mathcal{W}_{1}=\mathcal{H}(E)$, where $E(z):=$ $e^{-i z}=\cos z-i \sin z$. This is a de Branges space of finite and positive exponential type, and with $1 \in \operatorname{Assoc}_{\mathcal{P}} \mathcal{W}_{1}$.

We define a function $E_{+}=A_{+}-i B_{+}$by the relations

$$
A_{+}\left(z^{2}\right)=\cos z, \quad B_{+}\left(z^{2}\right)=z \sin z .
$$

Then $E_{+} \in \mathcal{H B}$ and $1 \in \operatorname{Assoc} \mathcal{H}\left(E_{+}\right)$; see Remark 2.13, (ii). Moreover, clearly, $\mathcal{H}\left(E_{+}\right)$ is of finite and positive $\sqrt{r}$-type, and the zeros of $A_{+}$are precisely the numbers $a_{k}:=$ $\pi^{2}\left(k-\frac{1}{2}\right)^{2}, k \in \mathbb{N}$.

The sequence $\left(a_{k}\right)_{k \in \mathbb{N}}$ has $\sqrt{r}$-density $\frac{1}{\pi^{2}}$. Since we assume that $\lambda(r)=O(r)$, we have $\sqrt{\lambda(r)}=O(\sqrt{r})$. By Lemma 3.4, we can choose a monotone nondecreasing sequence $\left(\gamma_{k}\right)_{k \in \mathbb{N}}$ of positive real numbers so that the sequence $\left(\gamma_{k} a_{k}\right)_{k \in \mathbb{N}}$ has finite and positive upper $\sqrt{\lambda(r)}$-density.

Clearly, $E_{+}$satisfies the assumptions (a) and (b). Therefore, we may apply Lemma 3.3 to obtain a function $\tilde{E}_{+}=\tilde{A}_{+}-i \tilde{B}_{+} \in \mathcal{H B}$ with $1 \in \operatorname{Assoc} \mathcal{H}\left(\tilde{E}_{+}\right), \tilde{B}_{+}(0)=0$, and $\tilde{A}_{+}(z)=\prod_{k=1}^{\infty}\left(1-\frac{z}{\gamma_{k} a_{k}}\right)$. By [L, I. Lehrsatz 17], the function $\tilde{A}_{+}$has finite and positive $\sqrt{\lambda(r)}$-type.

We choose $\tilde{C}_{+}, \tilde{D}_{+}$as in Remark 2.7 (and satisfying (2.8)), and put

$$
\tilde{W}_{+}:=\left(\begin{array}{ll}
\tilde{A}_{+} & \tilde{B}_{+} \\
\tilde{C}_{+} & \tilde{D}_{+}
\end{array}\right) .
$$

By the properties of the class $\mathcal{M}_{0}$, we have $\tilde{A}_{+}+i \tilde{C}_{+} \in \mathcal{H B}$ and $\tilde{C}_{+}(0)=0$. Since the zeros of $\tilde{A}_{+}$and $\tilde{C}_{+}$should interlace and all zeros of $\tilde{A}_{+}$are in $(0, \infty)$, it follows that all zeros of $\tilde{C}_{+}$are in $[0, \infty)$. We also have $\tilde{D}_{+}+i \tilde{C}_{+} \in \mathcal{H B}$, and thus the zeros of $\tilde{D}_{+}$and 
$\tilde{C}_{+}$interlace. Consequently, there are two possibilities:

(i) all zeros of $\tilde{D}_{+}$are in $(0, \infty)$;

(ii) there exists a unique $a<0$ such that $\tilde{D}_{+}(a)=0$.

In the case (i) the function $q=-\frac{\tilde{D}_{+}}{\tilde{B}_{+}}$is in the class $\mathcal{N}_{0}$, does not vanish on $(-\infty, 0)$, and has a pole at the zero. Hence, $q \in \mathcal{S}$ by Remark 2.12. We conclude that

$$
\lim _{x \rightarrow-\infty} \frac{\tilde{D}_{+}(x)}{\tilde{B}_{+}(x)} \in \mathbb{R} .
$$

By Remark 2.13, (iii), this implies that $1 \in \operatorname{Assoc} \mathcal{H}(\tilde{E})$ where $\tilde{E}$ is defined by

$$
\tilde{E}(z):=\tilde{A}_{+}\left(z^{2}\right)-\frac{i}{z} \tilde{B}_{+}\left(z^{2}\right)
$$

In the case (ii) we make use of the transformation $\mathfrak{w}_{a}$ introduced in Subsection 2d. Put

$$
\hat{W}_{+}=\left(\begin{array}{ll}
\hat{A}_{+} & \hat{B}_{+} \\
\hat{C}_{+} & \hat{D}_{+}
\end{array}\right):=\mathfrak{w}_{a}\left(\tilde{W}_{+}\right) .
$$

A computation shows that

$$
\hat{W}_{+}(z)=\left(\begin{array}{cc}
-\tilde{B}_{+}(a) \tilde{C}_{+}(z+a) & -\tilde{B}_{+}(a) \tilde{D}_{+}(z+a) \\
-\tilde{C}_{+}(a) \tilde{A}_{+}(z+a)+\tilde{A}_{+}(a) \tilde{C}_{+}(z+a) & -\tilde{C}_{+}(a) \tilde{B}_{+}(z+a)+\tilde{A}_{+}(a) \tilde{D}_{+}(z+a)
\end{array}\right) .
$$

Hence, we have

and

$$
\frac{\hat{D}_{+}(z)}{\hat{B}_{+}(z)}=\frac{\tilde{C}_{+}(a)}{\tilde{B}_{+}(a)} \frac{\tilde{B}_{+}(z+a)}{\tilde{D}_{+}(z+a)}-\frac{\tilde{A}_{+}(a)}{\tilde{B}_{+}(a)}
$$

$$
-\frac{\hat{A}_{+}(z)}{\hat{B}_{+}(z)}=-\frac{\tilde{C}_{+}(z+a)}{\tilde{D}_{+}(z+a)} .
$$

By Remark 2.12, the functions $-\frac{\hat{A}_{+}}{\hat{B}_{+}}$and $\frac{\tilde{B}_{+}(z+a)}{\tilde{D}_{+}(z+a)}$ belong to the Stieltjes class. Thus,

$$
\lim _{y \rightarrow+\infty} \frac{1}{y} \operatorname{Im} \frac{\hat{D}_{+}(i y)}{\hat{B}_{+}(i y)}=0 \quad \text { and } \quad \lim _{x \rightarrow-\infty} \frac{\hat{D}_{+}(x)}{\hat{B}_{+}(x)} \in \mathbb{R} .
$$

By Remark 2.13, (iii), we have $1 \in \operatorname{Assoc} \mathcal{H}(\hat{E})$, where

$$
\hat{E}(z):=\hat{A}_{+}\left(z^{2}\right)-\frac{i}{z} \hat{B}_{+}\left(z^{2}\right)
$$

Clearly, $\hat{E}$ is of finite and positive $\lambda$-type.

3.5. Remark. By using the construction of Remark 3.2, (iii), for $\lambda$ growing sufficiently slowly, it can be shown that the space $\mathcal{H}$ in Theorem 3.1 can be chosen in such a way that the domain of the multiplication operator in $\mathcal{H}$ is dense in $\mathcal{H}$.

Now, from Theorem 3.1 we can deduce the existence of de Branges spaces with subspaces of smaller growth.

3.6. Theorem. Let $\lambda, \lambda_{1}$ be growth functions with

$$
\lambda_{1}(r)=o(\lambda(r)) \quad \text { and } \quad \lambda(r)=O(r) .
$$

Then there exists a de Branges space $\mathcal{H}$ of finite and positive $\lambda$-type that has a subspace $\mathcal{L} \in \operatorname{Sub} \mathcal{H}$ of finite and positive $\lambda_{1}$-type. 
Proof. By Theorem 3.1, we can choose $W, W_{1} \in \mathcal{M}_{0}$ such that $\sigma_{W_{1}}^{\lambda_{1}}, \sigma_{W}^{\lambda} \in(0, \infty)$. Then $\sigma_{W_{1}}^{\lambda}=0$ and

whence

$$
\sigma_{W}^{\lambda} \leq \sigma_{W W_{1}}^{\lambda} \leq \sigma_{W}^{\lambda}+\sigma_{W_{1}}^{\lambda}=\sigma_{W}^{\lambda}
$$

$$
\sigma_{\mathfrak{v}\left(W W_{1}\right)}^{\lambda}=\sigma_{W W_{1}}^{\lambda}=\sigma_{W}^{\lambda} \in(0, \infty) .
$$

Consider the de Branges space $\mathcal{H}:=\mathcal{H}\left(E_{\mathfrak{v}\left(W W_{1}\right)}\right)$. Then, as we have just established, $\mathcal{H}$ is of finite and positive $\lambda$-type. Since $\mathfrak{v}\left(W W_{1}\right)=\mathfrak{v}\left(W_{1}\right) \mathfrak{v}(W)$, there exists $\mathcal{L} \in \operatorname{Sub} \mathcal{H}$ such that $\mathcal{L}=\mathcal{H}\left(E_{\mathfrak{v}\left(W_{1}\right)}\right)$ as sets. However,

$$
\sigma_{\mathcal{L}}^{\lambda_{1}}=\sigma_{\mathfrak{v}\left(W_{1}\right)}^{\lambda_{1}}=\sigma_{W_{1}}^{\lambda_{1}} \in(0, \infty)
$$

3.7. Remark. The following statement shows that the condition $\lambda(r)=O(r)$ in Theorem 3.6 is natural. Let $\lambda, \lambda_{1}$ be growth functions, and assume that

$$
\lambda_{1}(r)=o(\lambda(r)) \text { and } r=o(\lambda(r)) .
$$

Moreover, let $\mathcal{H}$ be a de Branges space of finite and positive $\lambda$-type. Then no nonzero subspace $\mathcal{L} \in \operatorname{Sub} \mathcal{H}$ can be of finite $\lambda_{1}$-type.

To see this, we recall that, by [KW3, Theorem 3.10], if $r=O(\mu(r))$ and a de Branges space contains one nonzero function of finite $\mu$-type, then it is itself of finite $\mu$-type. Applying this with

$$
\mu(r):=\max \left\{\lambda_{1}(r), r\right\}
$$

yields the desired conclusion.

\section{§4. A CONDition FOR NONEXistence of SUbSPaCES With SMALL GROWTH}

In this section we show that the existence of subspaces of a given de Branges space $\mathcal{H}$ that have smaller growth than $\mathcal{H}$ is by no means the generic situation. To be more precise, we show that if $\mathcal{H}$ is written as $\mathcal{H}(E)$ and the function $E$ has maximal admissible growth along the real axis, then the space $\mathcal{H}$ cannot contain infinite-dimensional subspaces of smaller growth.

4.1. Theorem. Let $\lambda$ and $\lambda_{1}$ be growth functions with $\lambda_{1}(r)=o(\lambda(r))$, and let $\mathcal{H}$ be $a$ de Branges space of finite and positive $\lambda$-type. Assume that for one (and hence for all) functions $E \in \mathcal{H B}$ with $\mathcal{H}=\mathcal{H}(E)$ we have $\log |E(x)| \asymp \lambda(|x|), x \in \mathbb{R}$. Then no infinite-dimensional subspace $\mathcal{L} \in \mathrm{Sub} \mathcal{H}$ is of finite $\lambda_{1}$-type.

Proof. Suppose that an infinite-dimensional de Branges subspace $\mathcal{H}\left(E_{1}\right)$ of $\mathcal{H}(E)$ has finite $\lambda_{1}$-type. Then

$$
\lim _{|x| \rightarrow \infty}\left|E_{1}(x) / E(x)\right|=0 .
$$

On the other hand, we have

$$
\|F\|_{E_{1}}=\|F\|_{E}, \quad F \in \mathcal{H}\left(E_{1}\right) .
$$

Recall that the reproducing kernel $K_{1}(\zeta, \cdot)$ of the space $\mathcal{H}\left(E_{1}\right)$ corresponding to the point $\zeta \in \mathbb{C}$ is of the form

$$
K_{1}(\zeta, z)=\frac{E(z) E^{\#}(\bar{\zeta})-E(\bar{\zeta}) E^{\#}(z)}{2 \pi i(\bar{\zeta}-z)} .
$$

We shall show that there exists a sequence $x_{n} \in \mathbb{R},\left|x_{n}\right| \rightarrow \infty$, such that

$$
\left\|K_{1}\left(x_{n}, \cdot\right)\right\|_{E}=o\left(\left\|K_{1}\left(x_{n}, \cdot\right)\right\|_{E_{1}}\right), \quad n \rightarrow \infty,
$$

which contradicts (4.1). 
Let $R>0$ and $x \in \mathbb{R}$. We have

$$
\left\|K_{1}(x, \cdot)\right\|_{E}^{2}=\int_{|t| \leq R}\left|\frac{K_{1}(x, t)}{E(t)}\right|^{2} d t+\int_{|t|>R}\left|\frac{K_{1}(x, t)}{E_{1}(t)}\right|^{2}\left|\frac{E_{1}(t)}{E(t)}\right|^{2} d t .
$$

Choosing $R$ sufficiently large, we can make the last summand smaller than $\varepsilon\left\|K_{1}(x, \cdot)\right\|_{E_{1}}^{2}$ for any given $\varepsilon>0$ and for all $x \in \mathbb{R}$. Thus, it suffices to show that for a fixed $R$ there exists a sequence $\left\{x_{n}\right\}$ such that

$$
\left\|K_{1}\left(x_{n}, \cdot\right)\right\|_{E_{1}}^{-2} \int_{|t| \leq R}\left|\frac{K_{1}\left(x_{n}, t\right)}{E(t)}\right|^{2} d t \rightarrow 0, \quad n \rightarrow \infty .
$$

Observe that $\left|K_{1}(x, t)\right| \leq \pi^{-1}|t-x|^{-1}|E(x) E(t)|$, and so

$$
\int_{|t| \leq R}\left|\frac{K_{1}(x, t)}{E(t)}\right|^{2} d t \leq C(R)\left|E_{1}(x)\right|^{2} x^{-2}, \quad|x|>R+1 .
$$

We also recall that

$$
\left\|K_{1}(x, \cdot)\right\|_{E_{1}}^{2}=\frac{\left|E_{1}(x)\right|^{2} \varphi_{1}^{\prime}(x)}{2 \pi}
$$

where $\varphi_{1}$ is the so-called phase function for $E_{1}$. Moreover,

$$
\varphi_{1}^{\prime}(x)=a+\sum_{n} \frac{\left|\operatorname{Im} z_{n}\right|}{\left|x-z_{n}\right|^{2}}
$$

where the $z_{n} \in \mathbb{C}^{-}$are the zeros of $E_{1}$ and $a \geq 0$ is the parameter in the factor $e^{-i a z}$ of the standard factorization of the Hermite-Biehler functions ([L]; see also [B1, KW2]).

To complete the proof, we need to show that

$$
\liminf _{|x| \rightarrow \infty} x^{-2}\left(\varphi_{1}^{\prime}(x)\right)^{-1}=0 .
$$

If $a>0$, then (4.2) is trivial. Otherwise, $E_{1}$ has infinitely many zeros, because we assumed the subspace $\mathcal{H}\left(E_{1}\right)$ to be infinite dimensional. Put $x_{n}=\operatorname{Re} z_{n}+\operatorname{sign}\left(\operatorname{Re} z_{n}\right)\left|\operatorname{Im} z_{n}\right|$. Then $\left|x_{n}\right| \rightarrow \infty$ and

$$
\varphi_{1}^{\prime}\left(x_{n}\right) \geq\left|2 \operatorname{Im} z_{n}\right|^{-1} \geq\left|2 x_{n}\right|^{-1} .
$$

Thus, we have $x_{n}^{2} \varphi^{\prime}\left(x_{n}\right) \rightarrow \infty$, which proves (4.2).

\section{REFERENCES}

[ADRS] D. Alpay, A. Dijksma, J. Rovnyak, and H. de Snoo, Schur functions, operator colligations, and reproducing kernel Pontryagin spaces, Oper. Theory Adv. Appl., vol. 96, Birkhäuser Verlag, Basel, 1997. MR1465432 (2000a:47024)

[B1] A. D. Baranov, The Bernstein inequality in the de Branges spaces and embedding theorems, Trudy S.-Peterburg. Mat. Obshch. 9 (2001), 23-53; English transl., Amer. Math. Soc. Transl. Ser. 2, vol. 209, Amer. Math. Soc., Providence, RI, 2003, pp. 21-49. MR2018371 (2004m:30043)

[B2] , Polynomials in the de Branges spaces of entire functions, Ark. Mat. 44 (2006), no. 1, 16-38. MR2237209

[BP1] C. Berg and H. Pedersen, On the order and type of the entire functions associated with an indeterminate Hamburger moment problem, Ark. Mat. 32 (1994), 1-11. MR1277917 (95i:30027)

[BP2] _ Nevanlinna matrices of entire functions, Math. Nachr. 171 (1995), $29-52$. MR1316350 (96a:47028)

[BP3] L L L L L L L inithmic order and type of indeterminate moment problems (with an appendix by W. Hayman), Difference Equations, Special Functions, and Applications (Munich, July 25-30, 2005) (to appear). 
[dB] L. de Branges, Hilbert spaces of entire functions, Prentice-Hall, Inc., Englewood Cliffs, NJ, 1968. MR0229011 (37:4590)

[DK1] H. Dym and H. McKean, Application of de Branges spaces of integral functions to the prediction of stationary Gaussian processes, Illinois J. Math. 14 (1970), 299-343. MR0260013 $(41: 4642)$

[DK2] - Gaussian processes, function theory, and the inverse spectral problem, Probab. Math. Statist., vol. 31, Acad. Press, New York-London, 1976. MR0448523 (56:6829)

[GM] L. Golinskii and I. Mikhailova, Hilbert spaces of entire functions as a J-theory subject, Topics in Interpolation Theory (Leipzig, 1994), Oper. Theory Adv. Appl., vol. 95, Birkhäuser, Basel, 1997, pp. 205-251. MR1473258 (98m:46031)

[KaK1] I. S. Kac and M. G. Krĕ̌n, R-functions - analytic functions mapping the upper halfplane into itself, F. V. Atkinson. Discrete and Continuous Boundary Problems, "Mir," Moscow, 1968, Supplement I to the Russian ed., pp. 629-647; English transl., Amer. Math. Soc. Transl. (2), vol. 103, Amer. Math. Soc., Providence, RI, 1974, pp. 1-18. MR0243149 (39:4473)

[KaK2] _ On the spectral functions of the string, F. V. Atkinson. Discrete and Continuous Boundary Problems, "Mir," Moscow, 1968, Supplement II to the Russian ed., pp. 648-737; English transl., Amer. Math. Soc. Transl. (2), vol. 103, Amer. Math. Soc., Providence, RI, 1974, pp. 19-102. MR0243149 (39:4473)

[KWW1] M. Kaltenbäck, H. Winkler, and H. Woracek, Singularities of generalized strings, Operator Theory and Indefinite Inner Product Spaces, Oper. Theory Adv. Appl., vol. 163, Birkhäuser, Basel, 2006, pp. 191-248. MR2215864 (2007f:47040)

[KWW2] - De Branges spaces of entire functions symmetric about the origin, Integral Equations Operator Theory 56 (2006), no. 4, 483-509. MR2284712

[KW1] M. Kaltenbäck and H. Woracek, Pontryagin spaces of entire functions. I, Integral Equations Operator Theory 33 (1999), 34-97. MR1664343 (2000a:46039)

[KW2] , Pólya class theory for Hermite-Biehler functions of finite order, J. London Math. Soc. (2) 68 (2003), no. 2, 338-354. MR1994686 (2004e:30042)

[KW3] De Branges spaces of exponential type: General theory of growth, Acta Sci. Math. (Szeged) 71 (2005), no. 1-2, 231-284. MR2160366 (2006c:30031)

[K1] M. G. Krein, A contribution to the theory of entire functions of exponential type, Izv. Akad. Nauk SSSR Ser. Mat. 11 (1947), no. 4, 309-326. (Russian) MR0022252 (9:179e)

[K2] On the indeterminate case of the Sturm-Liouville boundary problem in the interval $(0, \infty)$, Izv. Akad. Nauk SSSR Ser. Mat. 16 (1952), no. 4, 293-324. (Russian) MR0052004 $(14: 558 \mathrm{~g})$

[KL] M. G. Krein and H. Langer, Über einige Fortsetzungsprobleme, die eng mit der Theorie hermitescher Operatoren im Raume $\Pi_{\kappa}$ zusammenhängen. I. Einige Funktionenklassen und ihre Darstellungen, Math. Nachr. 77 (1977), 187-236. MR0461188 (57:1173)

[LG] P. Lelong and L. Gruman, Entire functions of several complex variables, Grundlehren Math. Wiss., Bd. 282, Springer-Verlag, Berlin, 1986. MR0837659 (87j:32001)

[L] B. Levin, Nullstellenverteilung ganzer Funktionen, Math. Lehrbücher Monogr. II. Abt. Math. Monogr., Bd. 14, Akademie-Verlag, Berlin, 1962. MR0150301 (27:302)

[Li] X.-J. Li, Hilbert spaces of entire functions and polynomials orthogonal on the unit circle, Methods Appl. Anal. 1 (1994), no. 1, 25-43. MR1260381 (95a:30004)

[OS] J. Ortega-Cerdà and K. Seip, Fourier frames, Ann. of Math. (2) 155 (2002), 789-806. MR1923965 (2003k:42055)

[P] G. Pick, Über die Beschränkungen analytischer Funktionen, welche durch vorgegebene Funktionswerte bewirkt werden, Math. Ann. 77 (1916), 7-23. MR1511844

[PW] V. Pivovarchik and H. Woracek, Shifted Hermite-Biehler functions and their applications, Integral Equations Operator Theory 57 (2007), no. 1, 101-126. MR2294277

[Re] C. Remling, Schrödinger operators and de Branges spaces, J. Funct. Anal. 196 (2002), no. 2, 323-394. MR1943095 (2003j:47055)

[RosR] M. Rosenblum and J. Rovnyak, Topics in Hardy classes and univalent functions, Birkhäuser Verlag, Basel, 1994. MR1307384 (97a:30047)

[RR] J. Rovnyak and V. Rovnyak, Sonine spaces of entire functions, J. Math. Anal. Appl. 27 (1969), 68-100. MR0243333 (39:4655)

[Ru] L. A. Rubel, Entire and meromorphic functions, Springer-Verlag, New York, 1996. MR1383095 (97c:30001) 
[Wi] H. Winkler, On transformations of canonical systems, Operator Theory and Boundary Eigenvalue Problems (Vienna, 1993), Oper. Theory Adv. Appl., vol. 80, Birkhäuser, Basel, 1995, pp. 276-288. MR1362114 (96m:34155)

[W] H. Woracek, De Branges spaces of entire functions closed under forming difference quotients, Integral Equations Operator Theory 37 (2000), no. 2, 238-249. MR1769812 (2001g:46058)

Department of Mathematics and Mechanics, St. Petersburg State University, UniverSitetskil Prospect 28, Stary I Peterhof, St. Petersburg 198504, Russia

E-mail address: antonbaranov@netscape.net

Institute of Mathematics, Royal Institute of Technology (KTH), SE-100, 44, Stockholm, SWEDEN

Current address: Institut für Analysis und Scientific Computing, Technische Universität Wien, Wiedner Hauptstr. 8-10/101, A-1040, Wien, Austria

E-mail address: harald.woracek@tuwien.ac.at

Received 22/APR/2006

Originally published in English 